TITLE:

\title{
Development of methodology to optimize management of failed fuels in light water reactors
}

$\operatorname{AUTHOR}(S)$ :

Yamamoto, Yasunori; Morishita, Kazunori

\section{CITATION:}

Yamamoto, Yasunori ...[et al]. Development of methodology to optimize management of failed fuels in light water reactors. Journal of Nuclear Science and Technology 2014, 52 (5): 709-716

ISSUE DATE:

2014-11-17

URL:

http://hdl.handle.net/2433/200754

\section{RIGHT:}

This is an Accepted Manuscript of an article published by Taylor \& Francis in 'Journal of Nuclear Science and

Technology', available online: http://www.tandfonline.com/10.1080/00223131.2014.979901.; The full-text file will be made open to the public on 17 Nov 2015 in accordance with publisher's 'Terms and Conditions for Self-Archiving'; This is not the published version. Please cite only the published version.; この論文は出版社版でありません。引用の際には 出版社版をご確認ざ利用ください。 


\title{
ARTICLE
}

\section{Development of methodology to optimize management of failed fuels in light water reactors}

\author{
Yasunori Yamamoto ${ }^{a}$ and Kazunori Morishita ${ }^{b *}$ \\ ${ }^{a}$ Graduate School of Energy Science, Kyoto University, Gokasho, Uji, Kyoto 611-0011, \\ Japan; $\quad$ b Institute of Advanced Energy, Kyoto University, Gokasho, Uji, Kyoto 611-0011, \\ Japan
}

\section{Acknowledgements}

The authors would like to acknowledge the national research project on “Aging Management Program Development on System Safety of Nuclear Power Plants in Japan”, which is operated by Mitsubishi Research Institute, Inc., Japan, and sponsored by Nuclear Regulation Authority, Japan, for providing us with an opportunity to exchange useful information and knowledge related to this research.

\begin{abstract}
Fuel cladding is one of the key components in a fission reactor that confines radioactive materials inside a fuel tube. During reactor operation, however, cladding is sometimes breached, and radioactive materials leak from the fuel pellet into the coolant water through the breach. The primary coolant water is therefore monitored so that any leak is quickly detected; coolant water is periodically sampled, and the concentration of radioactive iodine 131 (I-131), for example, is measured. Depending on the measured leakage concentration, the faulty fuel assembly with leaking rod is removed from the reactor and replaced immediately or at the next refueling. In the present study, an effort has been made to develop a methodology to optimize the management for replacement of faulty fuel assemblies due to
\end{abstract}


cladding failures using measured leakage concentration. A model numerical equation is proposed to describe the time evolution of an increase in I-131 concentration due to cladding failures and is then solved using the Monte Carlo method as a function of sampling rate. Our results indicate that, to achieve rationalized management of failed fuels, higher resolution to detect a small amount of I-131 is not necessarily required, but more frequent sampling is favorable.

Keywords: fuel cladding; Monte Carlo method; iodine; safety assessment; leak; fuel failure

*Corresponding author. Email:morishita@iae.kyoto-u.ac.jp 


\section{Introduction}

A fuel cladding tube in a fission reactor separates the radioactive fuel pellet from the rest of the reactor system and has an important role as a barrier against the leak of radioactive materials to the exterior. By the presence of the tube, the concentration of radioactive materials in the primary coolant water remains suppressed at a sufficiently low level to mitigate the consequences of so-called pipe rupture accidents, leading to an effective reduction in radiation exposure risks for the public. Although the fabrication of fuel cladding has been accomplished by high-quality processes to prevent the formation of manufacturing defects, cladding failures can still occur due to such phenomena during operation as pellet-cladding interaction (PCI), fretting corrosion, excessive corrosion, stress corrosion cracking (SCC), crud deposition, and embrittlement due to hydrides [1-4]. Once cladding failures occur, radioactive materials leak from the fuel pellet into the primary coolant water through a cladding breach. To detect those leaks, the coolant water is periodically monitored, typically a few times a week, and the primary coolant water is kept under surveillance to confirm the concentration of radioactive iodine 131 (I-131) in the sampled water within the limiting conditions for operation (LCO) with regard to fuel leaks. The threshold maximum concentration satisfying LCO is defined by the safety regulations in Japan and determined to minimize radiation exposure risks for the public.

When the LCO regulation is violated, the reactor is immediately shut down to remove and replace the faulty fuel assembly with leaking rod. In practice, however, the faulty fuel assembly is replaced at a much lower concentration than the LCO threshold, which is a consequence of practical reactor operations that obey a different shutdown rule than the LCO regulation. This shutdown rule is decided in advanced by reactor operators themselves, which is usually more conservative than the LCO. As there is currently a growing tendency for reactor operators in Japan to obey more conservative operator rules than the LCO, the number of unscheduled immediate reactor shutdowns has recently increased, as shown in Figure 1, in 
which the annual number of operational responses to fuel leaks is represented for commercial pressurized water reactors (PWRs) and boiling water reactors (BWRs) in Japan [5].

These conservative judgments initially seem to be favorable in terms of safety, but, when considering a material's integrity and, moreover, overall reactor safety, such an immoderate conservative judgment is not always favorable because the materials in a reactor are damaged by additional thermal and mechanical loadings during unscheduled shutdown processes. Thus, fuel leaks due to cladding failures require different levels of operator intervention that depend on the degree of severity of cladding failures or leaks. When the leak is not so severe, no immediate response is required because the risks caused by immediate reactor shutdown are considered to be larger than those caused by leaving failed fuels in place. In this case, the faulty fuel assembly with leaking rod should not be removed until the next refueling. When the leak is significant, however, an immediate reactor shutdown is required to remove the faulty fuel assembly.

The problem is that the degree of severity of cladding failures is not always sufficiently obvious for the operator to choose one of the two above interventions because severity varies over time; the severity is not always clear, except with the opportunities of water sampling for monitoring, although many efforts have been made to establish a method to predict the future behavior of fuel claddings during use in a reactor [6-11]. Therefore, actual operator interventions become too conservative unless an appropriate prediction method is well established.

In the present study, a new attempt has been made to optimize the management for the replacement of faulty fuel assemblies due to cladding failures, which is discussed in terms of the critical concentration of I-131 in the primary coolant water as well as the rate of water sampling to monitor I-131concentration. In section 2, the usual methodology for probabilistic risk assessment (PRA) [12] is introduced and applied to an issue of fuel management optimization. Additionally, a model numerical equation describing the time evolution of I-131 
concentration in the coolant water is proposed to be solved by the valuable Monte Carlo method. In section 3, some calculation results are shown as example cases, where the frequency of immediate reactor shutdown is evaluated as a risk caused by variation in the severity of fuel failures. An optimized management for the replacement of faulty fuel assemblies with leaking rod is then discussed. Finally, a conclusion is provided in section 4.

\section{Procedure}

\subsection{Methodology of risk assessment}

PRA is a useful and comprehensive methodology to find the residual risks associated with a complex engineered system. PRA is often applied to the safety assessment of nuclear power plants, using the event-tree (ET) and fault-tree (FT) techniques for detail calculations. In the present study, a unique attempt was made using the concept of PRA to optimize the management of fuel replacement accompanying cladding failures. As an index indicating the optimized level of management, the frequency of unscheduled immediate reactor shutdowns was chosen; the unscheduled immediate shutdown is a type of fuel management that is performed to cope with fuel leaks due to cladding failures. In this section, the procedure of PRA is simply explained using an example of seismic PRA [12] for better understanding.

In the framework of conventional seismic PRA [12], such parameters as the core damage frequency (CDF) and containment failure frequency (CFF) are evaluated for reactor safety assessment, using ET and FT analyses that follow given accident scenarios with hazard and fragility curves, as shown in Figure 2 (a). The hazard curve shows the frequency of exceedance of seismic action as a hazard, which is in general a decreasing function of the peak ground acceleration (PGA), the magnitude of the hazard. This figure indicates that seismic actions with higher PGA occur less frequently. The fragility curve, however, exhibits 
the occurrence probability of the failure of instruments, devices, and components in a reactor, which is reasonably considered to be an increasing function of PGA.

We now consider cladding failures and the accompanying leaks of radioactive materials from the fuel pellet into the primary coolant water through a cladding breach. These events are very complicated and not easily described in detail due to the involvement of various phenomena, as explained above. An attempt to investigate the mechanisms of complicated events is very interesting in terms of materials science and fluid dynamics but is not the scope of the present study. Apart from those complexities and difficulties, let us consider a simple model of leak events with a reasonable assumption that a larger amount of leakage occurs less frequently. Two parameters, $F$ and $L$, are introduced here to describe leak events, where $F$ is the number of leak events occurring in unit time, and $L$ is the amount of leakage per event with units of Bq (Becquerel)/event. From this assumption, $F$ should be a decreasing function of $L$. The product $F \times L$ indicates a leak rate, $R$, with units of $\mathrm{Bq} / \mathrm{s}$, which may be an index reflecting the states of cladding materials and the surrounding environment. Figure 3 shows one example of relationships between $F$ and $L$, where $R$ is a constant.

Next, let us compare the seismic hazard curve in Figure 2 (a) with the equivalent hazard curve in Figure 3 that represents the occurrence frequency of leak events. The two curves seem to be very similar. Based on this similarity, we have drawn the schematic diagram represented in Figure 2 (b), in which the occurrence frequency of fuel leaks due to cladding failures and the occurrence probability of unscheduled immediate reactor shutdowns due to fuel leaks are shown to correspond to the hazard and fragility curves in Figure 2 (a), respectively. From this correspondence, a leak event may be regarded as a hazard against the stable operation of a reactor. The amount of leakage per event may indicate the hazard magnitude, which may correspond to PGA in the seismic PRA. If this analogy is correct, we may finally obtain the shutdown frequency curve from our equivalent hazard and fragility curves, which could correspond to the core damage frequency curve in Figure 2 (a). 
However, the present effort to apply PRA methodology to our fuel leak issues would have difficulty in preparing the formalism of the evaluation. Namely, the equivalent fragility curve that represents the occurrence probability of unscheduled immediate reactor shutdowns is not easily drawn from the beginning as a function of the amount of leakage per event, although the conventional PRA method requires a fragility curve prior to making an assessment. The amount of leakage on a per-event basis indicates the amount of radioactive materials released at once from the fuel pellet into the primary coolant water through a cladding breach, which could depend on such parameters as the size and shape of a breach, the ambient temperature, the coolant water pressure, and their gradients. Unfortunately, however, the amount of leakage per event cannot serve an appropriate role as a criterion index to judge unscheduled immediate reactor shutdowns because reactor shutdowns are judged by the concentration of radioactive materials accumulated so far in the coolant water. Therefore, in the present study, instead of ET and FT analyses along with fragility curves, we used the Monte Carlo technique. With this technique, the equivalent fragility curve is obtained later as an output of the Monte Carlo calculations rather than used as an input for the calculations.

\subsection{Monte Carlo model}

The concentration of radioactive I-131 in the primary coolant water is considered to be determined by a balance among the rate of leaks from fuel rods to the coolant water, the rate of radioactive decay, and the rate of reduction by purification at the water cleanup system in a reactor. The time derivative of concentration is thus expressed by the following equation [13]:

$$
\frac{\mathrm{d} C}{\mathrm{~d} t}=\frac{R}{V}-\lambda C-\frac{Q}{V} C
$$

where $C$ is the $\mathrm{I}-131$ concentration in the primary coolant water in $\mathrm{Bq} / \mathrm{m}^{3}$, $t$ is the time in seconds, $V$ is the volume of the primary coolant water system in $\mathrm{m}^{3}, \lambda$ is the radioactive decay 
constant of $\mathrm{I}-131$ in $\mathrm{s}^{-1}, R$ is the leak rate described above, and $Q$ is the rate of flow in the water cleanup system in $\mathrm{m}^{3} / \mathrm{s}$. Note that the purification efficiency of the cleanup system is effectively involved in parameter $Q$. The parameter numbers used for the present calculations are listed in Table 1. Assuming that $R$ is a constant, $C$ is obtained by integrating Equation (1), as follows:

$$
C(t)=\frac{R}{\lambda V+Q}\left\{1-\exp \left[-\left(\lambda+\frac{Q}{V}\right) \cdot t\right]\right\} \quad
$$

Figure 4 shows the time evolution of I-131 concentration obtained by equation (2). As time evolves, the concentration approaches an asymptotic value of $R /(\lambda V+Q)$ that the concentration never theoretically exceeded.

In practice, however, the concentration does not exhibit such a smooth and expectable curve because the cladding breach may be created by several different, complicated mechanisms described above, and, in addition, the crack initiation and propagation behavior during cladding failures is stochastic in essence. Moreover, leak behavior from the pellet to the primary coolant water through a breach is also stochastic, which may depend on not only the state of a breach (size and shape) but also reactor operating conditions [14]. To describe these stochastic behaviors, the Monte Carlo method could be an appropriate method and is applied here when a discretized form of Equation (1) is numerically integrated. The Monte Carlo method used in the present study is the same as that often used in materials modeling [15], and the time step used for numerical integrations was provided by the $n$-fold method [16], as $\Delta t=-\ln (r) / F$, where $r$ is a random number between 0 and 1 . Figure 5 shows an example of our calculation results, in which the time evolution of I-131 concentration is shown for cases with high and low $F$. The average behaviors of time evolution are the same; however, the fluctuations of concentrations are quite different for the two cases. The concentration sometimes exceeds even the asymptotic 
value due to the existence of large fluctuations created by the Monte Carlo technique. The fluctuations are much greater for the case of low $F$ than that of high $F$. This difference may lead to a clear difference in fuel management, as described below.

To model the management of fuel replacement, a critical value of I-131 concentration is defined here, as represented by the horizontal dashed lines in Figure 5. Using the critical value, a judgment was made of whether or not to perform the fuel replacement. Namely, when the monitored temporal concentration is detected to excess the critical value, the reactor is judged to be shut down immediately to replace the faulty fuel assemblies with leaking rod. No constraints were imposed on the critical value in the present study, although it should primarily be identical to the value that is determined to follow the operator's own rule or the LCO regulation, as described above.

Furthermore, in the model, water sampling for monitoring I-131 concentration was assumed to be performed intermittently and periodically, as is currently done at actual nuclear power plants in Japan. If the monitoring is performed intermittently, there is a possibility of detection failure. Namely, even if the concentration in the coolant water is higher than the critical value, such an abnormal concentration can escape from detection when the state happens between water samplings. Such a possibility becomes higher when the sampling rate is lower. Despite intermittent monitoring with potential detection failures, when a higher concentration than the critical value is successfully detected at the opportunity of water sampling, the reactor is judged to be shut down, and the concentration is immediately forced to be zero in the model. This operation implies that fuel replacement is made at that time. Figure 6 shows an example of the model fuel management designed here, where water sampling is conducted once a week, and the critical concentration is given as indicated in the figure. The figure clearly shows that the concentration is forced to be zero when concentration exceeds the critical value at the opportunity of water sampling.

From our discussion so far, model fuel management is eventually characterized by two 
control parameters, the critical concentration value and the water sampling rate. For each set of the two control parameters, 1000 trials of the Monte Carlo calculations were performed to obtain the statistics. The number of fuel replacements during one cycle operation period was then counted, with one cycle period set to 13 months, which is equivalent to an operation period for commercial reactors in Japan. Finally, the frequency of unscheduled immediate reactor shutdowns was obtained as a function of the two control parameters.

\section{Results and Discussion}

Figure 7 is a double logarithmic plot of the equivalent hazard curve for fuel leaks, showing the occurrence frequency of leak events as a function of the amount of I-131 leakage per event. In the present study, we make the appropriate assumption that events with larger amounts of leakage occur less frequently. Using these data in the plot as input, Monte Carlo simulations were performed to obtain the time evolution of I-131 concentrations as well as the number of immediate reactor shutdowns that is performed to cope with fuel leaks, as a function of the two parameters, the critical concentration and the sampling rate.

Figure 8 shows the shutdown frequency, which is defined as the average number of immediate reactor shutdowns during 13 months. Here, the leak rate, $R$, used for calculations is varied, as shown in the figure, but the critical concentration and sampling rate are fixed at $1.3 \times 10^{9} \mathrm{~Bq} / \mathrm{m}^{3}$ and $1.65 \times 10^{-6} \mathrm{~s}^{-1}$, respectively. As shown in the figure, the shutdown frequency has a peak that increases with the leak rate, $R$. Fuel management requiring an immediate reactor shutdown becomes more frequent when the leak rate is larger, as easily expected. Furthermore, as shown in the figure, the amount of leakage per event at the peak position does not highly depend on the leak rate, $R$.

Figure 9 shows the equivalent fragility curves that represent the probability of immediate reactor shutdown as a function of the amount of I-131 leakage per event. Different 
from the original PRA technique, these equivalent fragility curves are obtained as a result of calculations, as mentioned above. As shown in the figure, the probability of immediate reactor shutdown increases with the amount of I-131 leakage per event, which may indicate that the amount of leakage on a per event basis is well correlated with the amount of leakage accumulated in the coolant water. Because the amount of leakage per event is considered to be related to such breach attributes as size and shape, suppression of the formation of relatively large breaches is an appropriate primary measure to efficiently reduce the number of unscheduled reactor shutdowns.

From closer inspection of the curve, the probability of immediate reactor shutdown exhibits a sudden rise at approximately $1 \times 10^{13} \mathrm{~Bq} /$ event in this case, independent of the leak rate, $R$. The amount of I-131 leakage per event at the sudden rise in the figure would seem to effectively be a threshold value for immediate reactor shutdown. When the amount of leakage per event exceeds the threshold, an immediate reactor shutdown is performed with a high probability to cope with fuel leaks, as shown in Figure 9, although those leak events occur infrequently, as is shown in Figure 7. The threshold value thus defined does not depend much on the leak rate, $R$, again, and would be consistent with the amount of I-131 leakage per event at the peak position of Figure 8 .

To obtain useful knowledge to realize optimized fuel management, the sensitivity of the two operational parameters, critical concentration and sampling rate, to the calculation results was investigated. Figure $\mathbf{1 0}$ shows the calculated shutdown frequency curves for a fixed value of $R=3 \times 10^{6} \mathrm{~Bq} / \mathrm{s}$ as a function of the critical value of I-131 concentration to be used to judge whether or not immediate reactor shutdown occurs. Taking the lower critical value would seem to suggest that fuel management becomes more conservative, where earlier fuel replacements are performed for the purpose of pursuing reactor safety. Most likely, this expectation would not be inaccurate; unfortunately, however, it may not be so effective, as clearly shown in the figure. Even if the critical concentration adopts a lower value, only the 
threshold value is sensitive and becomes lower, indicating that an object to be treated is expanded only to smaller breaches. Smaller breaches may have smaller impact on the future development of leak events in terms of fracture mechanics; therefore, fuel management using a relatively low critical concentration is considered to be less effective.

Our Monte Carlo calculations also evaluated shutdown frequency as a function of sampling rate. Figure $\mathbf{1 1}$ indicates the frequency of unscheduled immediate reactor shutdowns for a fixed value of leak rate, $R=3 \times 10^{6} \mathrm{~Bq} / \mathrm{s}$, when the time interval of intermittent monitoring is changed from one day to one month. The time interval is the reciprocal of the sampling rate. With increased time interval, the frequency of immediate reactor shutdowns decreases as shown in the figure because, when the time interval becomes longer, the opportunity to find abnormal concentrations of I-131 in the coolant water is reduced. To clarify this point, additional calculations were performed to obtain the frequency of immediate reactor shutdowns when monitoring is continuously performed. By this monitoring, the concentration of I-131 in the coolant water can always be monitored; therefore, an unscheduled immediate reactor shutdown can be performed without fail for all events that require fuel replacement management. Figure $\mathbf{1 2}$ is a plot of the difference of the frequency of immediate reactor shutdown obtained by intermittent monitoring and that by continuous monitoring. The figure actually represents the frequency of occurrence of such an event that fuel replacement is really required but unfortunately missed. As shown in the figure, the frequency curve thus defined also has a peak, and the missing events obviously increase with increased time interval.

Figure 11 also shows that the curves for a larger amount of I-131 leakage per event are very sensitive to a change in time interval, whereas the threshold is not influenced. This result may indicate that fuel management with a short time interval, i.e., frequent sampling, is an appropriate way to efficiently find fuel leaks through large breaches while maintaining the threshold. Thus, monitoring with more frequent sampling is more favorable. 


\section{Conclusion}

An attempt has been made to develop a methodology to optimize the fuel management for replacement of faulty fuel assemblies due to cladding failures, which was discussed in terms of such operational parameters as the critical concentration of I-131 leakage and the water sampling rate. The methodology was established within a similar framework as probabilistic risk assessment. A model numerical equation to describe the time evolution of I-131 concentration due to fuel leaks was proposed and then solved using the Monte Carlo method. The frequency of immediate reactor shutdown that is performed to cope with faulty fuel assemblies was used as an index indicating the optimized level of management and obtained as a function of the operational parameters. From the calculation results, we have finally concluded that, to achieve the optimized management of failed fuels, high resolution to detect a small amount of I-131 is not necessarily required, but frequent sampling is favorable. Further improvement is required for practical use of the model in actual fuel replacement management. Model validation and an appropriate comparison with experiments should be extensively performed as a future study. 


\section{References}

[1] Garzarolli F, Jan RV, Stehle H. [The main causes of fuel element failure in water-cooled power reactors]. Atomic Energy Rev. 1979; 17 1: 31-128.

[2] El-Adham K. [Fuel failure mechanisms in operating U.S. plants from 1981 to 1986]. J. Nucl. Safety. 1988; 29 4: 487-500.

[3] Yang RL. Meeting the challenge of managing nuclear fuel in a competitive environment. Proceedings of International Topical Meeting on Light water reactor fuel performance: American Nuclear Society; 1997; Oregon (Portland).

[4] Yamamoto Y, Morishita K, Iwakiri H, Kaneta Y. [Theoretical investigation of oxidation mechanism of fuel cladding in light-water reactor]. E-journal of advanced maintenance. 2013;5 1:1-6.

[5] Japan Nuclear Safety Institute. Nuclear Information Archives. <http://www.nucia.jp/>.

[6] Kim MS, Kim KT. [Simulation of release rate coefficients of fission products for debris-induced fuel failures]. Nuclear Engineering and Design. 2013; 255: 123-131.

[7] Iqbal MJ, Mirza NM, Mirza SM. [Stochastic simulation of fission product activity in primary coolant due to fuel rod failures in typical PWRs under power transients]. J. Nucl. Mat.. 2008; 372: 132-140.

[8] Lewis BJ, El-Jaby A, Higgs J, Thompson WT, Iglesias FC, Laidler R, Armstrong J, Stone R, Oduntan. [A model for predicting coolant activity behavior for fuel-failure monitoring analysis]. J. Nucl. Mat.. 2007; 366: 37-51.

[9] Andrews WS, Lewis BJ, Cox DS. [Artificial neural network models for volatile fission product release during severe accident conditions]. J. Nucl. Mat.. 1999; 270: 74-86.

[10] Chun MH, Tak NI, Lee SK. [Development of a computer code to estimate the fuel rod failure using primary coolant activities of operating PWRs]. Ann. Nucl. Energy. 1998; 25 10: 753-763.

[11] Koo YH, Sohn DS. [Release of unstable fission products from defective fuel rods to the 
coolant of a PWR]. J. Nucl. Mat. 1994; 209: 248-258.

[12] Sugino H, Iwabuchi Y, Nishio M, Tsutsumi H, Sakagami M, Ebisawa K. [Development of probabilistic methodology for evaluating tsunami risk on nuclear power plants]. The $14^{\text {th }}$ World Conference on Earthquake Engineering: 2008; Beijin (China).

[13] Nasumi Ishiwatari. [Release rate of iodine 131 from defective fuel in a water loop of nuclear reactor]. Journal of The Chemical Society of Japan. 1981; 6: 1021-1026 [in Japanese].

[14] International Atomic Energy Agency (IAEA). Fuel failure in water reactors: Causes and mitigation. Proceedings of a Technical Meeting: 2002; Bratislava (Slovakia). IAEA-TECDOC-1345.

[15] Yamamoto Y, Yoshimatsu J, Morishita K. [Damage rate dependence of defect cluster nucleation in tungsten during irradiation]. J. Nucl. Mat. 2013; 442: S773-S775.

[16] Battaile CC, Srolovitz DJ, Butler JE. [A kinetic Monte Carlo method for the atomistic-scale simulation of chemical vapor deposition: Application to diamond]. J. Appl. Phys. 1997; 82 (12): 6293-6300. 
Table 1 Parameter values used in the present calculations

\begin{tabular}{|l|l|}
\hline Leak rate, $R$ & $0.3 \times 10^{7} \mathrm{~Bq} / \mathrm{s} \sim 3.0 \times 10^{7} \mathrm{~Bq} / \mathrm{s}$ \\
\hline Decay constant, $\lambda$ & $9.964 \times 10^{-7} \mathrm{~s}^{-1}$ \\
\hline Flow rate, $Q$ & $2.14 \times 10^{-2} \mathrm{~m}^{3} / \mathrm{s}$ \\
\hline System volume, $V$ & $7570 \mathrm{~m}^{3}$ \\
\hline Critical concentration & $0.3 \times 10^{9} \mathrm{~Bq} / \mathrm{m}^{3} \sim 1.3 \times 10^{9} \mathrm{~Bq} / \mathrm{m}^{3}$ \\
\hline Sampling rate & $3.86 \times 10^{-7} \mathrm{~s}^{-1} \sim 1.16 \times 10^{-5} \mathrm{~s}^{-1}$ \\
& $($ Corresponding time interval: 1 month, 2 weeks, 1 week, 1day) \\
\hline
\end{tabular}




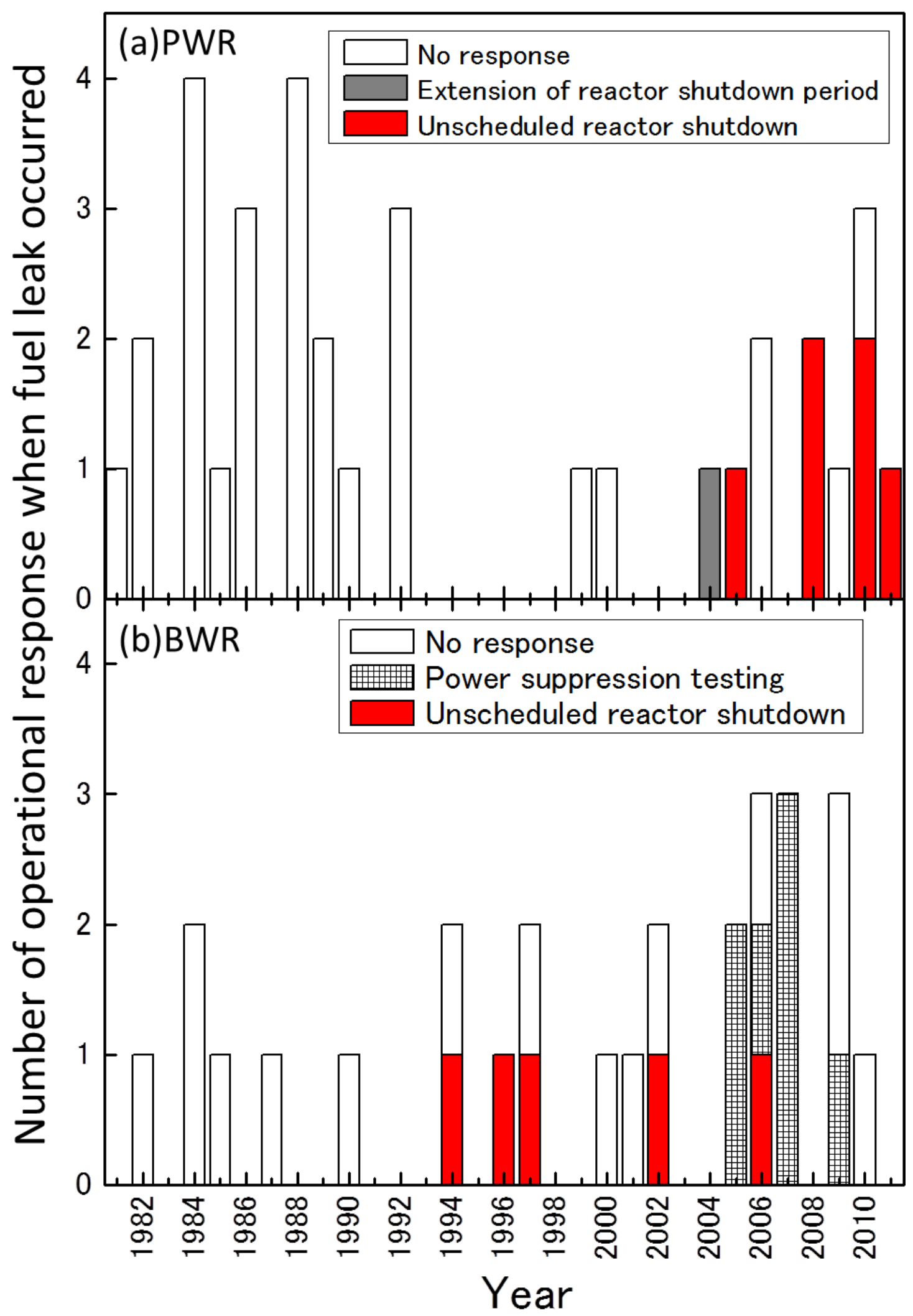

Fig.1 Number of operational response to fuel leaks per year conducted at (a) PWRs and (b) BWRs in Japan [5]. 

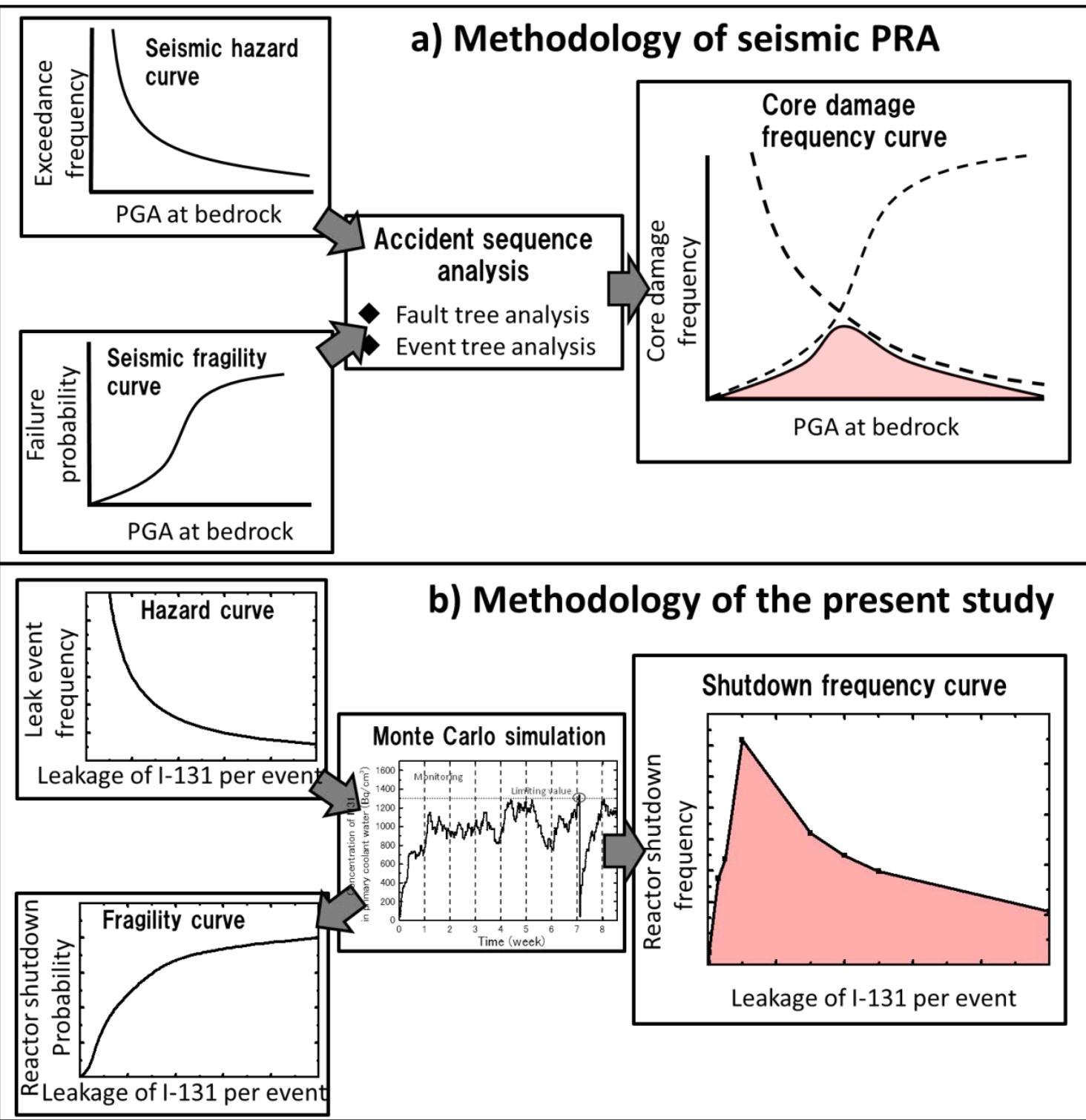

Fig.2 Schematic representations of the methodology of (a) seismic PRA [12] and (b) the present study. 


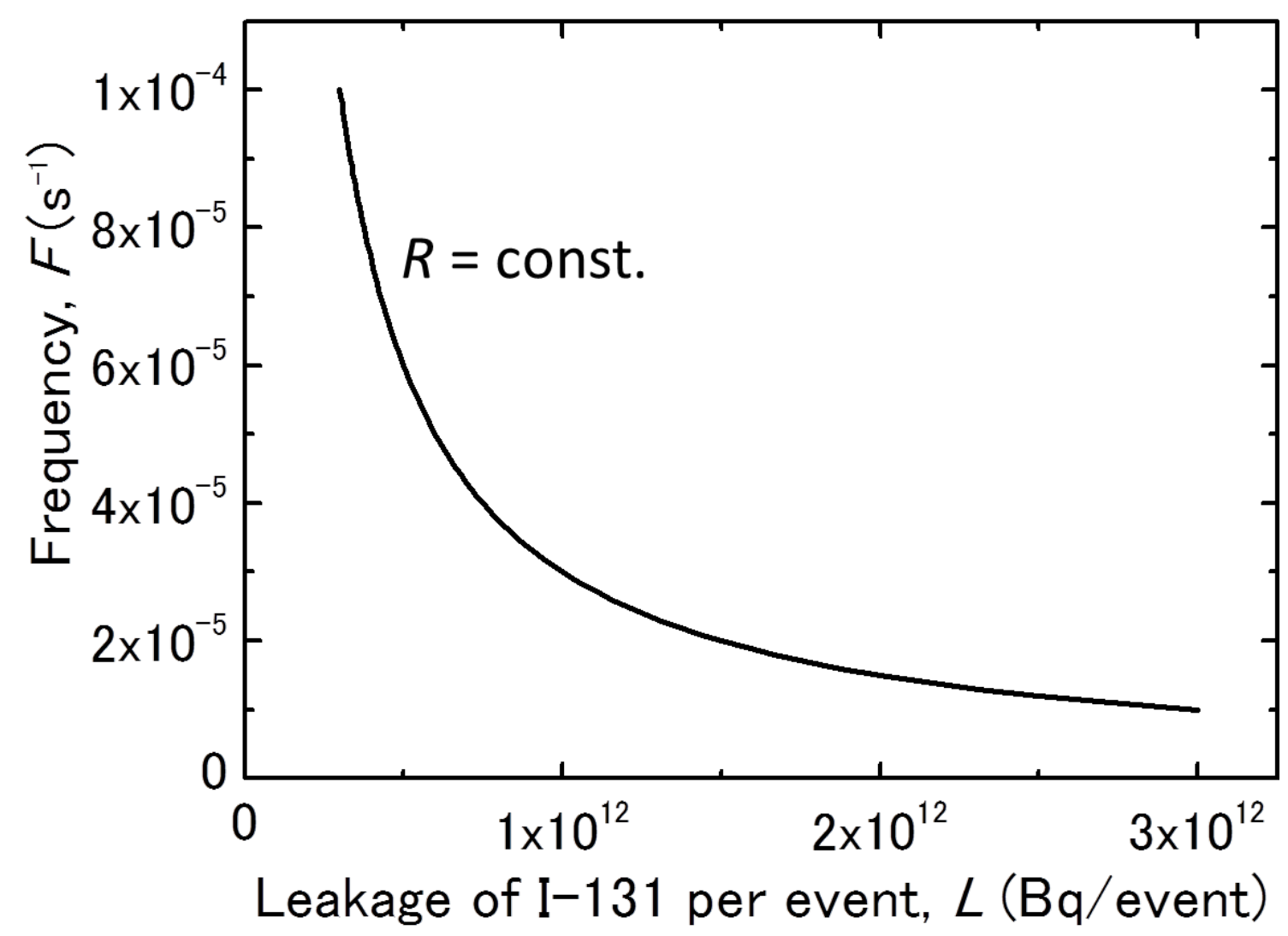

Fig.3 Equivalent hazard curve representing the occurrence frequency of leak events as a function of the leakage of I-131 per event. 


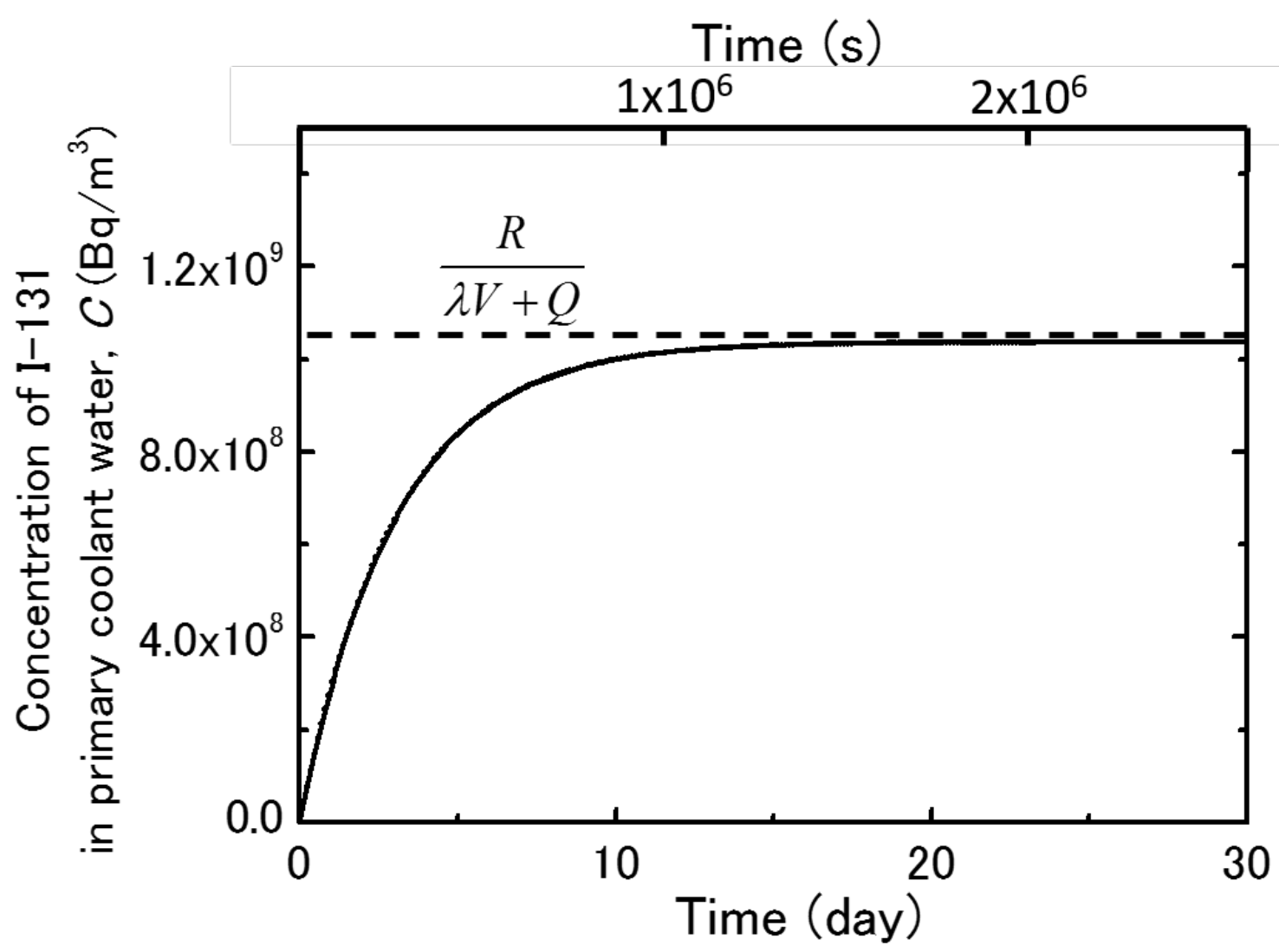

Fig.4 Time evolution of I-131 concentration in the primary coolant water. 


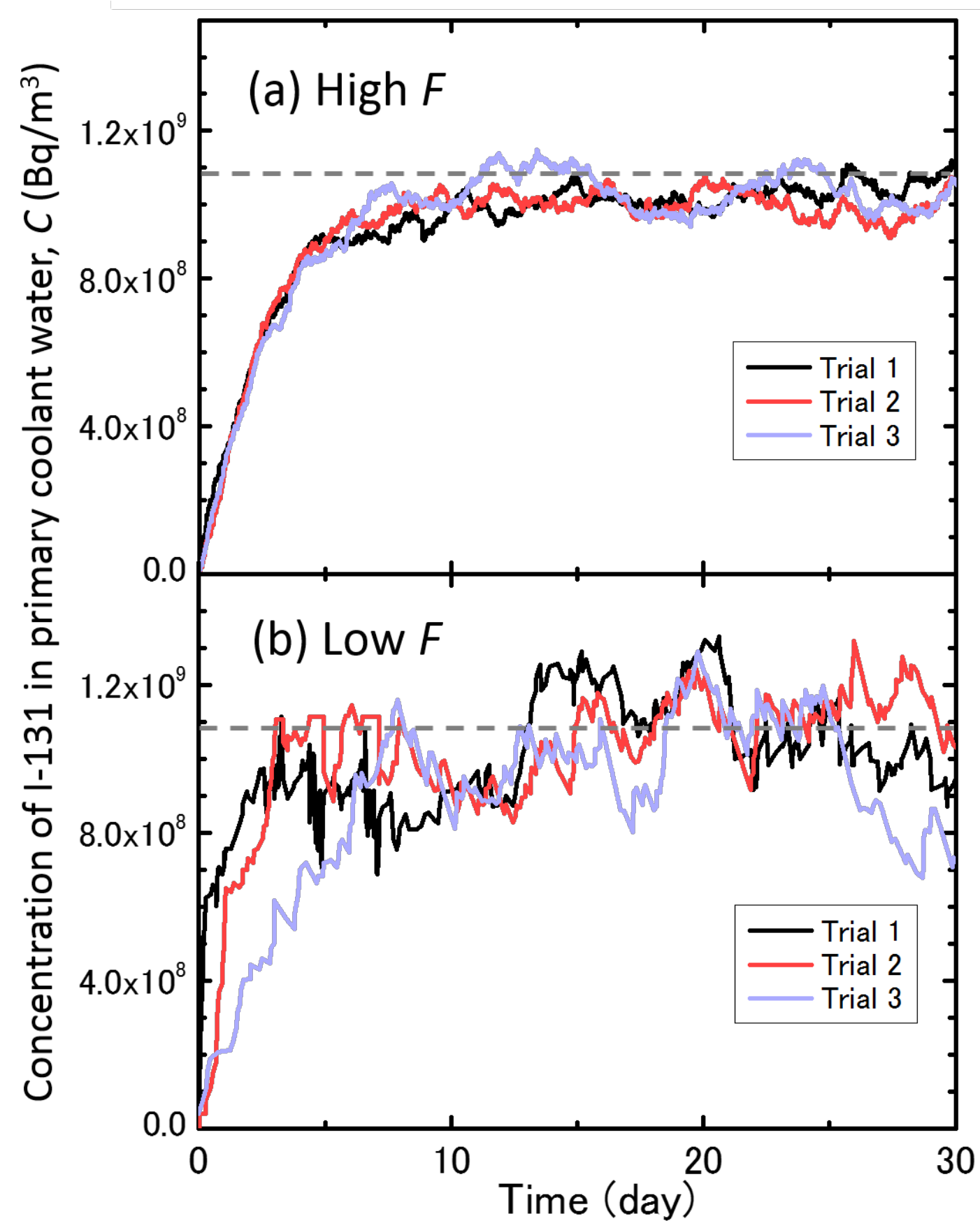

Fig.5 Time evolution of the concentration of I-131 in the primary coolant water for the cases of (a) high frequency condition and (b) low frequency condition. 


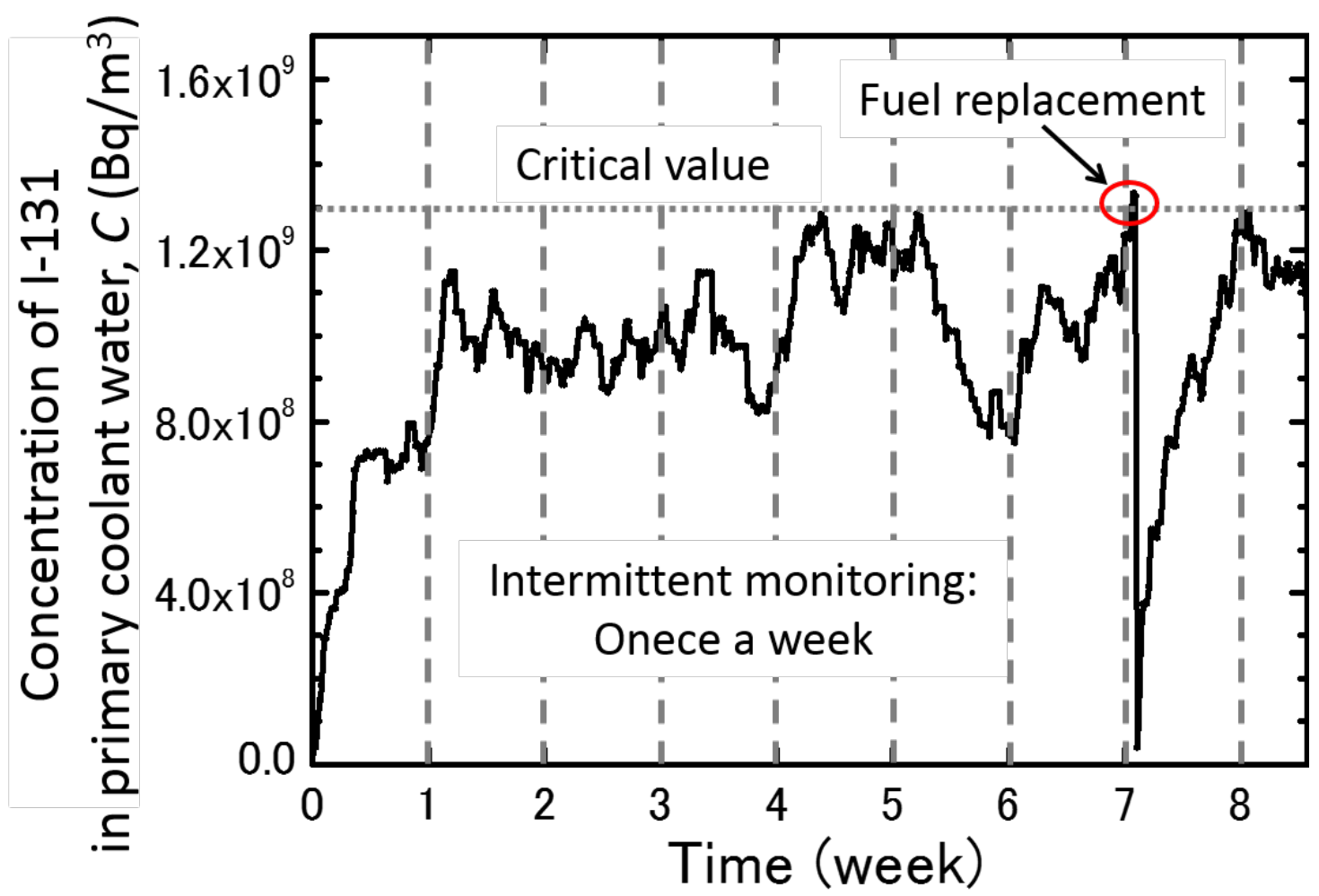

Fig.6 An example of calculations, which represents the time evolution of I-131 concentration when the water sampling is conducted once a week. The critical concentration is given as indicated by the horizontal dashed line. In this case, the fuel replacement is observed to be performed at just after 7 weeks, when the concentration is set to be zero, followed by a subsequent calculation trial. 


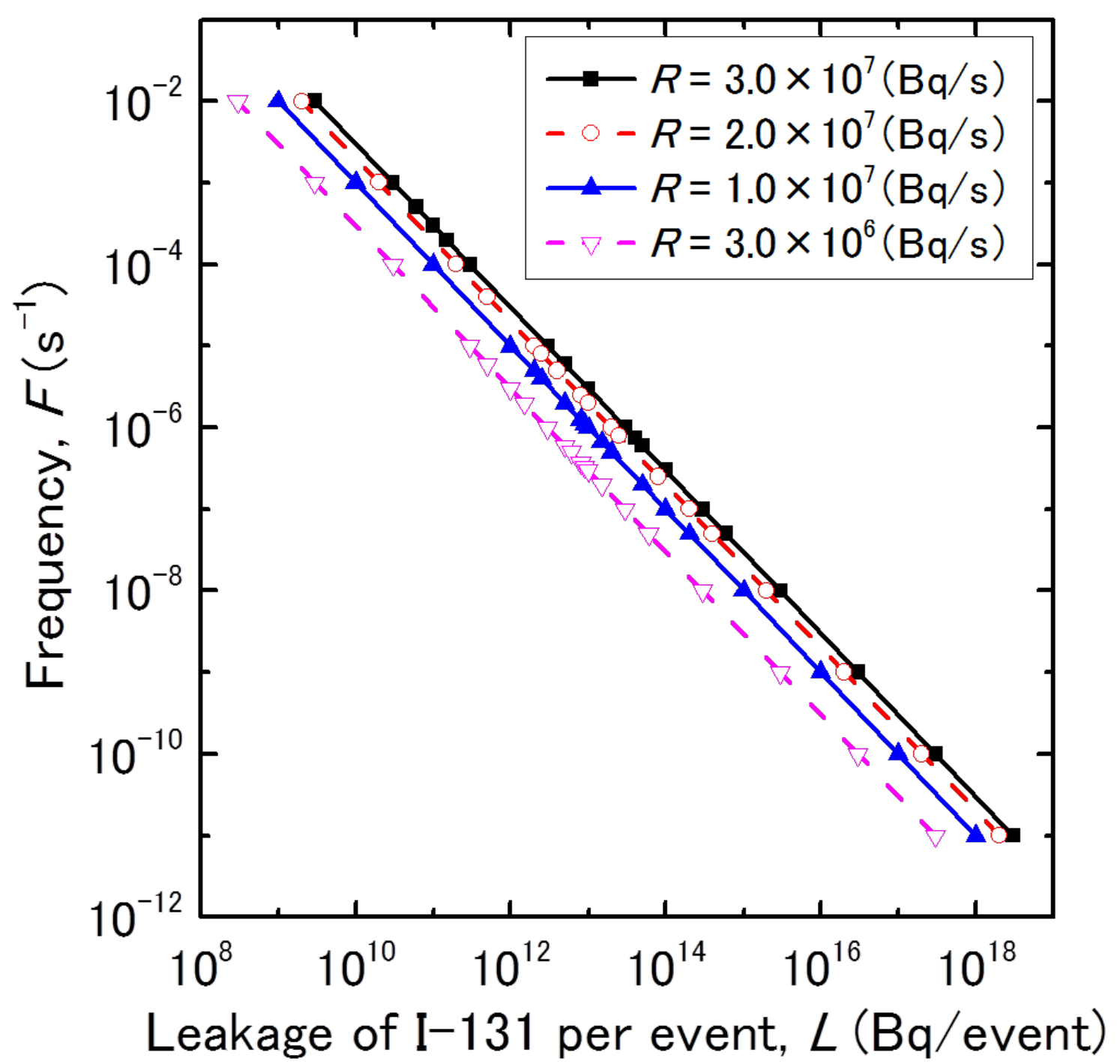

Fig.7 A double logarithmic plot of the equivalent hazard curve for fuel leaks. 


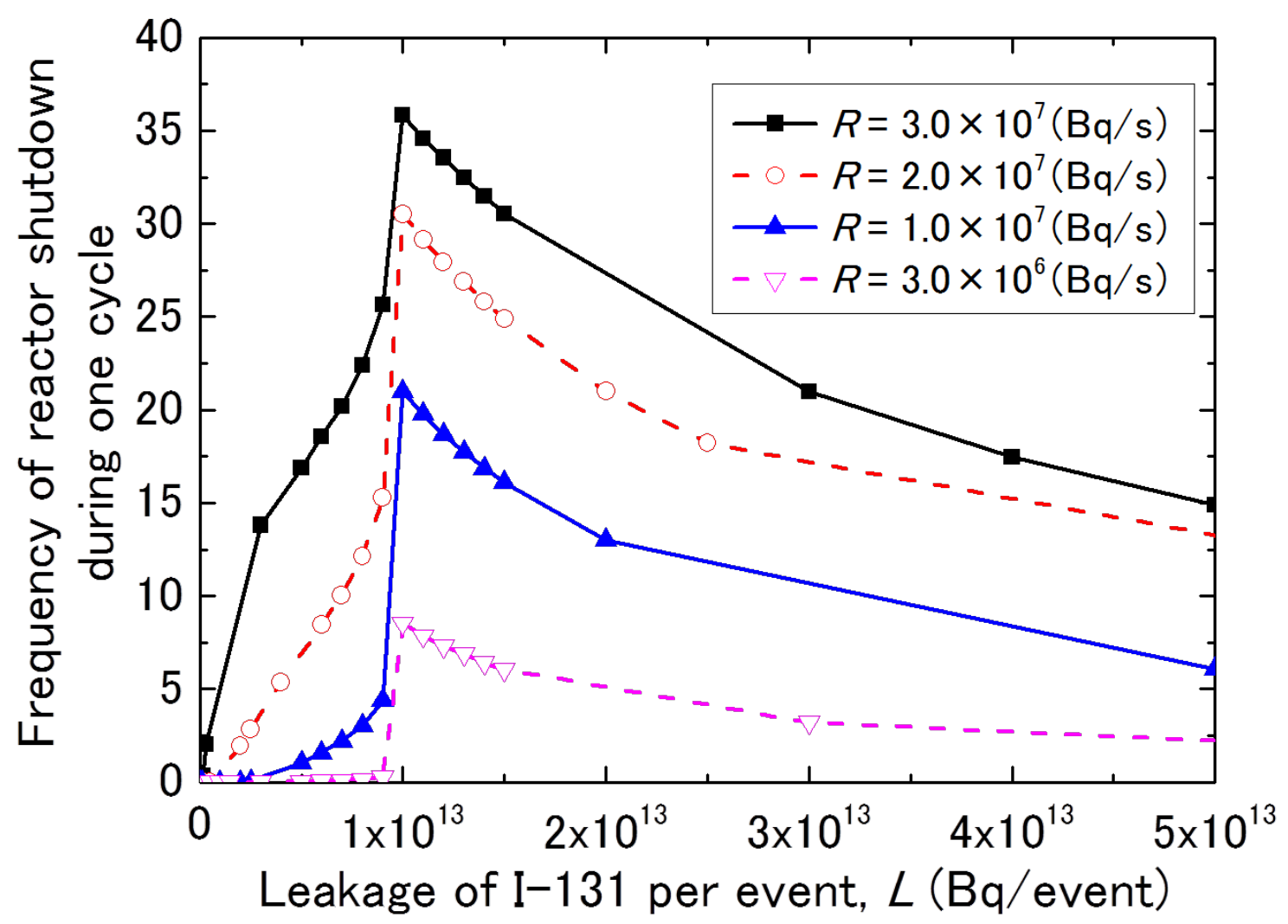

Fig.8 Frequency of unscheduled immediate reactor shutdown due to fuel leaks for various leak rates, $R$. 


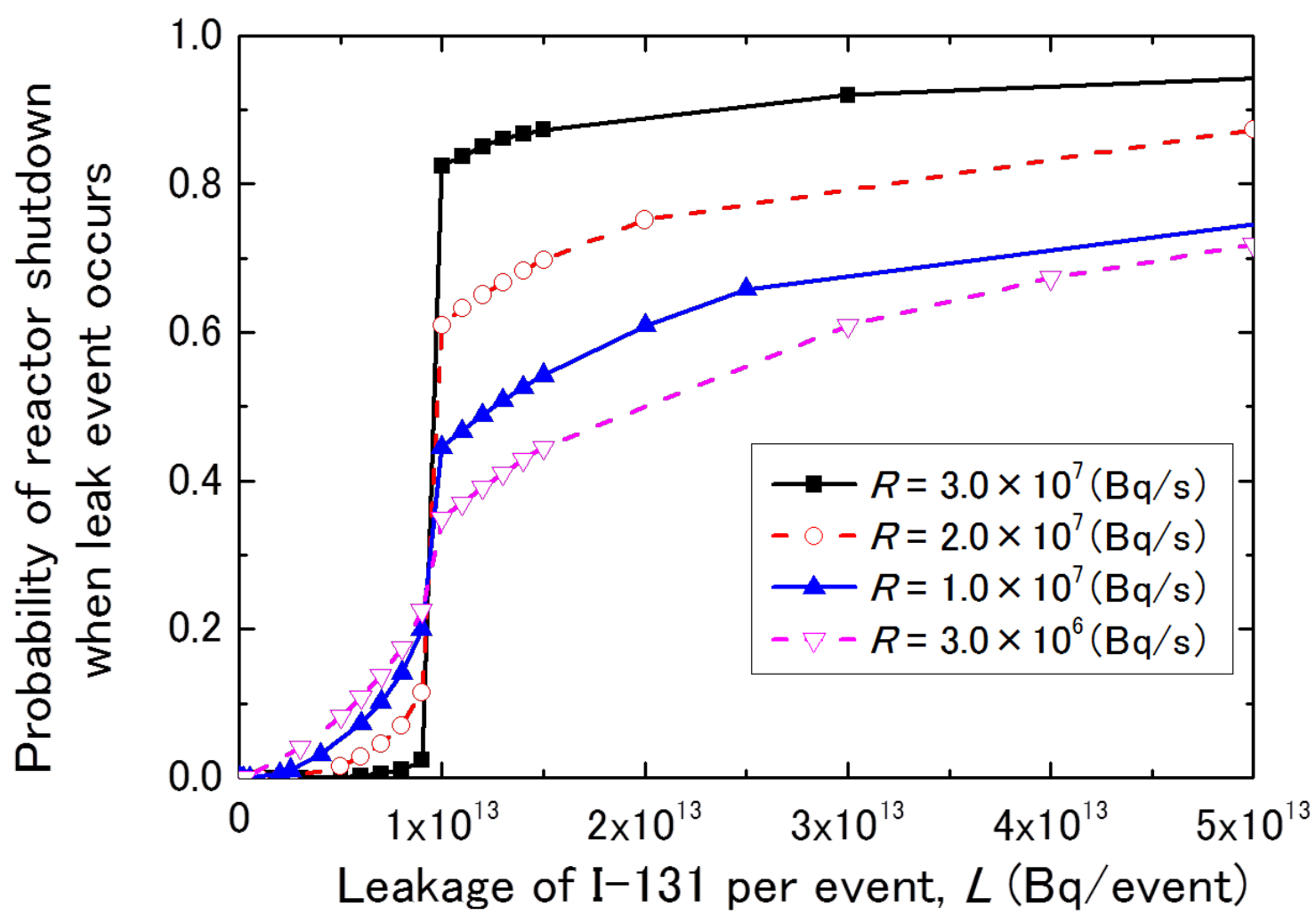

Fig.9 Equivalent fragility curve for fuel leaks obtained by the Monte Carlo calculations. The fuel leak event is considered to be almost a threshold phenomenon. 


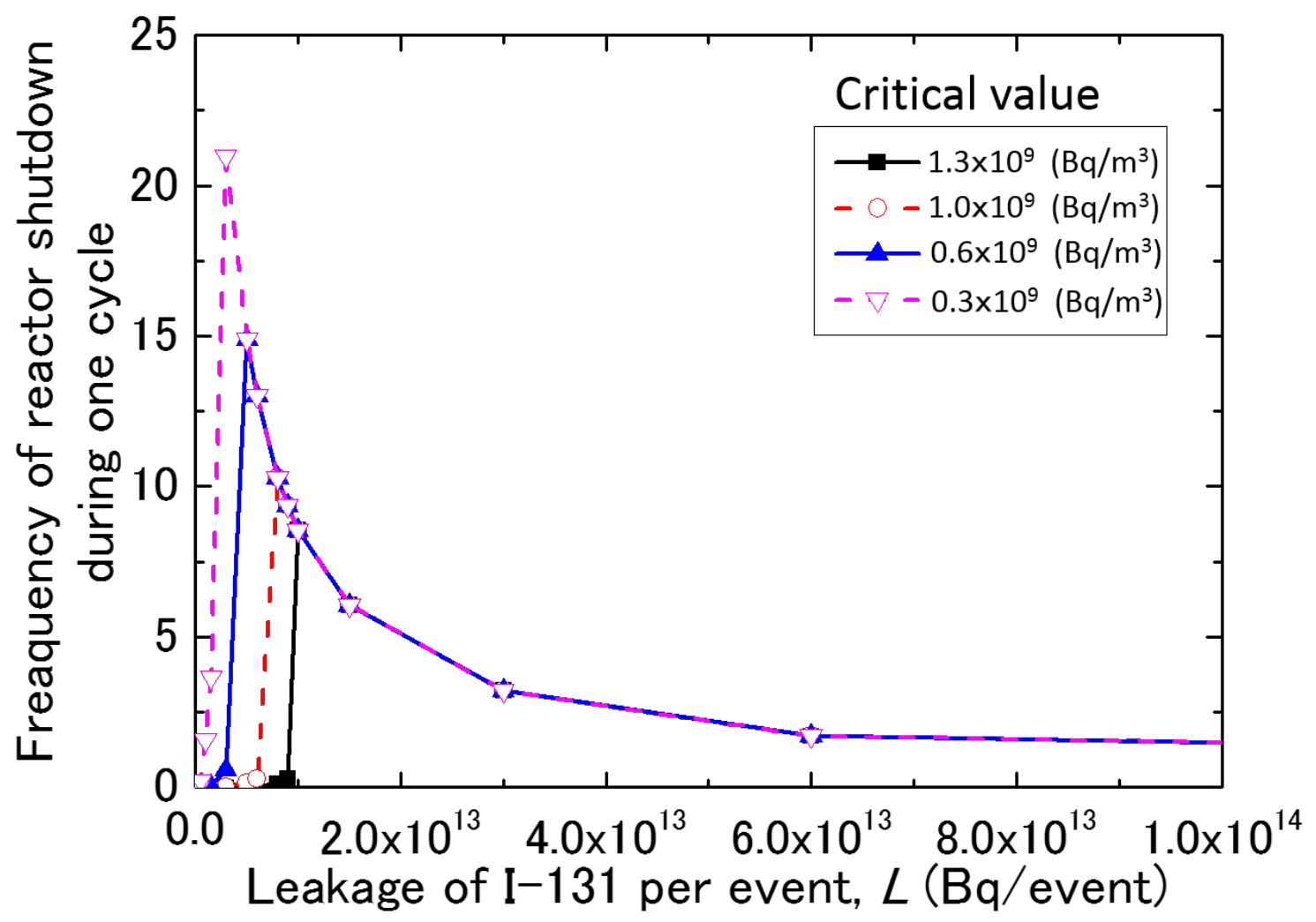

Fig.10 Shutdown frequency curves when the critical value of I-131 concentration was changed. 


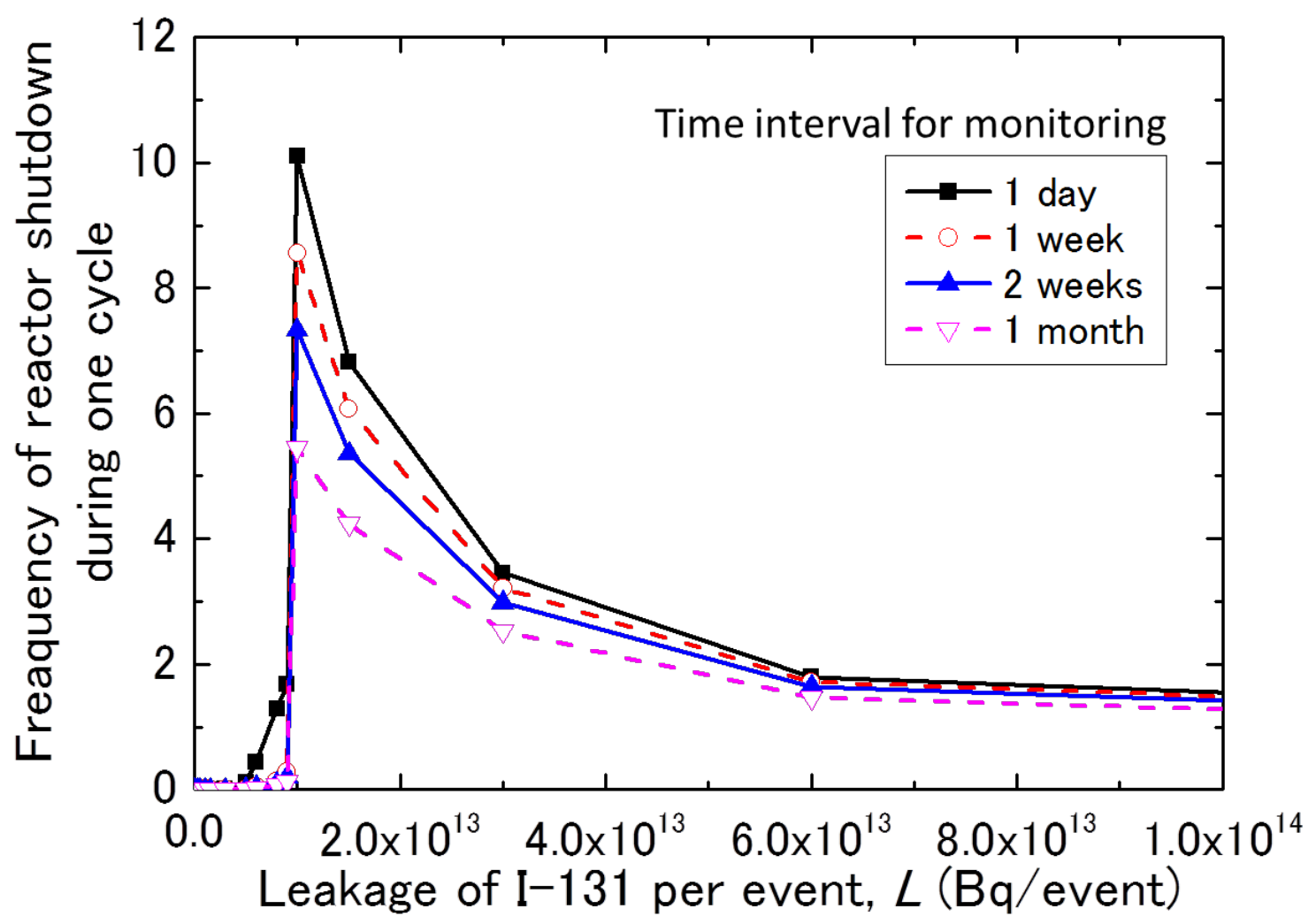

Fig. 11 Frequency of immediate reactor shutdown for a fixed value of the leak rate, $R=3 \times 10^{6} \mathrm{~Bq} / \mathrm{s}$, when the time interval of intermittent monitoring is changed from one day to one month. 


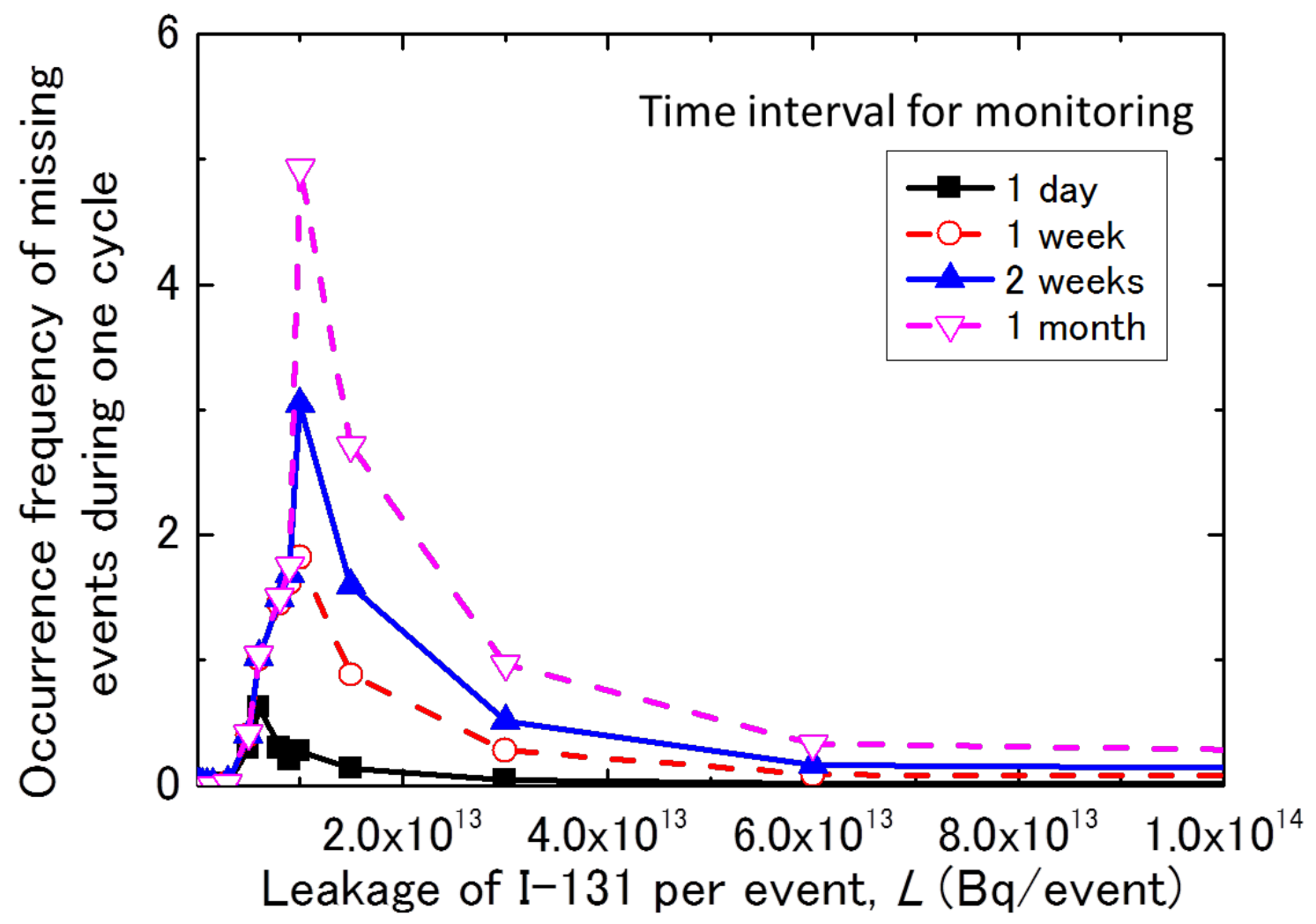

Fig. 12 Frequency of the occurrence of such an event that the fuel replacement is really required but unfortunately missed. 